\title{
AVALIAÇÃO DA SATISFAÇÃO DE USUÁRIOS CIRÚRGICOS DE HOSPITAIS DE ENSINO: ANÁLISE DA TANGIBILIDADE*
}

\author{
Maria Paula Custódio Silva ${ }^{1}$, Joyce Mara Gabriel Duarte ${ }^{2}$, Nara dos Santos Costa ${ }^{3}$, Andréia Silva Dutra \\ Tirones ${ }^{4}$, Ana Lúcia de Assis Simões ${ }^{5}$
}

\begin{abstract}
RESUMO: O objetivo do estudo foi avaliar a satisfação de clientes cirúrgicos de hospitais de ensino de Uberaba/ Minas Gerais, quanto à dimensão tangibilidade. Foram entrevistados 228 clientes, através de um questionário sociodemográfico e o instrumento baseado na escala SERVQUAL, cujos itens de 1 a 4 são relativos à dimensão tangibilidade. Os dados foram coletados entre julho de 2013 e fevereiro de 2014, e analisados através do programa estatístico Statistical Package for Social Sciences. A análise da expectativa e percepção em relação ao domínio tangibilidade evidenciou que os clientes esperam que o hospital apresente estrutura física conservada e moderna, que a equipe tenha uma aparência pessoal adequada ao ambiente e que haja informação disponível de fácil compreensão. Já as médias do gap variaram entre $-0,31(s=1,07)$ e $-0,05(s=0,75)$. A dimensão tangibilidade indicou insatisfação, ou seja, não atendeu as expectativas dos participantes em relação aos objetos tangíveis. DESCRITORES: Satisfação do paciente; Qualidade da assistência à saúde; Indicadores de qualidade em assistência à saúde.
\end{abstract}

\section{ASSESSMENT OF SATISFACTION AMONG SURGICAL CLIENTS OF TEACHING HOSPITALS: ANALYSIS OF TANGIBILITY}

\begin{abstract}
Abstract The present study aimed to assess the degree of satisfactedion of surgical clients of teaching hospitals in Uberaba/Minas Gerais, regarding the tangibility dimension. In the study, 228 clients were interviewed using a sociodemographic questionnaire and an instrument based on the multi-item scale SERVQUAL, whose items 1 to 4 concern the dimension tangibility. Data was collected between July 2013 and February 2014, and analyzed with the SPSS program. Analysis of expectation and perception regarding the tangibility domain showed that clients expect to be treated in a hospital equipped with a modern and adequate physical structure and whose workers maintain a professional well-groomed appearance and where easy-to-understand information is available. The mean values of the gap ranged between $-0.31(\mathrm{~s}=1.07)$ and $-0.05(\mathrm{~s}=0.75)$. The dimension tangibility revealed dissatisfaction, i.e. it did not meet the participants' expectations regarding tangible objects.

DESCRIPTORS: Patient satisfaction; Quality of health care; Quality indicators in health care.
\end{abstract}

\section{EVALUACIÓN DE SATISFACCIÓN DE PACIENTES QUIRÚRGICOS DE HOSPITALES DE ENSEÑANZA: ANÁLISIS DE TANGIBILIDAD}

RESUMEN: El objetivo del estudio fue evaluar la satisfacción de pacientes quirúrgicos de hospitales de enseñanza de Uberaba/Minas Gerais, respecto de la dimensión tangibilidad. Fueron entrevistados 228 pacientes, utilizando un cuestionario sociodemográfico y el instrumento basado en la escala SERVQUAL, cuyos ítems del 1 al 4 son relativos a la dimensión tangibilidad. Datos recolectados de julio 2013 a febrero 2014, analizados utilizando programa estadístico Statistical Package for Social Sciences. El análisis de la expectativa y percepción respecto del dominio tangibilidad evidenció que los pacientes esperan que el hospital presente estructura física conservada y moderna, que el equipo tenga la apariencia personal adecuada al ámbito y que exista información disponible de sencilla comprensión. Ya los promedios del gap variaron entre $-0,31(s=1,07)$ y $-0,05(s=0,75)$. La dimensión tangibilidad indicó insatisfacción, es decir, no satisfizo las expectativas de los participantes respecto de los objetos tangibles.

DESCRIPTORES: Satisfacción del Paciente; Calidad de la Atención de Salud; Indicadores de Calidad de la Atención de Salud.

\footnotetext{
*Artigo extraído da dissertação intitulada: "Cirurgias eletivas em hospitais públicos de uma cidade do interior de minas gerais: os cidadãos estão satisfeitos?". Universidade Federal do Triângulo Mineiro, 2014.
}

${ }^{1}$ Enfermeira. Residente em Enfermagem em Neonatologia. Universidade Federal do Triângulo Mineiro. Uberaba, MG, Brasil. ${ }^{2}$ Enfermeira. Doutoranda em Atenção à Saúde. Universidade Federal do Triângulo Mineiro. Uberaba, MG, Brasil.

${ }^{3}$ Enfermeira. Mestre em Atenção à Saúde. Docente na Escola Invicta. Uberaba, MG, Brasil.

${ }^{4}$ Enfermeira. Mestre em Atenção à Saúde. Enfermeira Assistencial na Unidade de Terapia Renal do Hospital de Clínicas da Universidade Federal do Triângulo Mineiro. Uberaba, MG, Brasil.

${ }^{5}$ Enfermeira. Doutora em Enfermagem. Docente de Enfermagem e do Programa de Pós-graduação em Atenção à Saúde da Universidade Federal do Triângulo Mineiro. Uberaba, MG, Brasil.

Autor Correspondente:

Maria Paula Custódio Silva

Universidade Federal do Triângulo Mineiro

Av. Frei Paulino, 410 - 38025180 - Uberaba, MG, Brasil

E-mail: mariaenf33@gmail.com
Recebido: 02/11/2016

Finalizado: 19/04/2017 


\section{- INTRODUÇÃO}

A avaliação dos serviços de saúde pela ótica do usuário é uma prática que vem se difundindo há algumas décadas, um importante indicador de qualidade nos serviços de saúde ${ }^{(1)}$. A qualidade de um serviço pode ser determinada pelo resultado da relação entre expectativas e percepções do usuário sobre o atendimento recebido ${ }^{(2)}$. Portanto, relação estabelecida entre o serviço, seja ele público ou privado, influenciará a percepção do usuário sobre a assistência prestada ${ }^{(1)}$.

A expectativa é o desejo ou necessidade dos usuários em relação aos serviços. Ela está relacionada à percepção sobre o atendimento oferecido e pode gerar satisfação ou insatisfação, refletindo a avaliação do usuário sobre a qualidade do serviço. A satisfação do usuário compreende uma resposta emocional, cognitiva e uma experiência de consumo, que ocorre em um tempo particular com base na experiência acumulada ${ }^{(3-4)}$.

A avaliação da qualidade dos serviços oferecidos, sob o olhar do usuário, permite que as empresas melhorem suas práticas, buscando atender às necessidades e direitos do usuário, além de contribuir para um atendimento mais humanizado. Nessa perspectiva, foi desenvolvido o instrumento SERVQUAL, baseado na expectativa e na percepção do consumidor sobre a qualidade do serviço ${ }^{(5)}$.

O instrumento é constituído por cinco dimensões: tangibilidade, confiabilidade, responsabilidade, segurança e empatia. A dimensão tangibilidade avalia a aparência do pessoal, instalações físicas, equipamentos, informática e informação ${ }^{(5)}$. Tangível é aquilo que se pode tanger ou tocar, ser palpável, sendo tangibilidade uma qualidade ou caráter de tangível ${ }^{(6)}$.

Ao encontro da Política Nacional de Humanização, o ambiente do serviço de saúde deve propiciar um atendimento acolhedor, resolutivo e humanizado, atendendo os princípios do Sistema Único de Saúde (SUS) de equidade, integralidade e universalidade. Para promover esse atendimento humanizado, o espaço onde o cuidado é oferecido desse ser limpo e organizado, com mobília adequada, comida saudável e de boa procedência, ventilação suficiente, água acessível, roupas e banheiros asseados. Essas questões chamadas por tecnologias duras que, quando bem estruturadas, conferem boa qualidade ao serviço $^{(7-9)}$.

Apesar de há tempos discutir-se a inclusão do usuário no processo de avaliação dos serviços de saúde, este ainda não é meio tão frequentemente utilizado. Tem se dado maior destaque a indicadores como índice de infecção hospitalar ou média de dias de internação, não considerando a satisfação do cliente $^{(10-11)}$.

Incluir o cliente externo no processo de saúde quer dizer também incluí-lo na sua avaliação. Na avaliação da qualidade, as expectativas e percepções têm papel fundamental, refletindo na avaliação da qualidade do serviço. A satisfação do cliente confere ações positivas, como adesão ao tratamento, continuidade em longo prazo e busca pela prevenção dos agravos ${ }^{(12)}$.

A avaliação da qualidade dos serviços de saúde colabora para realização de melhorias aos cidadãos, permitindo fortalecimento do SUS e de políticas públicas existentes, elaboração de novas propostas e identificação de fragilidades do sistema que os gestores desconhecem.

Realizadas as devidas considerações, depara-se com o problema que originou a necessidade de realização deste estudo: Avaliar a qualidade dos serviços oferecidos pelas instituições hospitalares prestadoras de assistência à saúde no SUS, em relação à tangibilidade, sob a perspectiva dos clientes cirúrgicos.

Assim, o objetivo desta pesquisa foi avaliar a satisfação dos clientes cirúrgicos de hospitais de ensino em uma cidade do interior de Minas Gerais (MG), na dimensão tangibilidade.

\section{METODOLOGIA}

Trata-se de uma pesquisa descritiva, observacional, longitudinal e prospectiva, utilizando abordagem quantitativa. Este estudo é recorte de um projeto maior intitulado: "Cirurgias eletivas em 
hospitais públicos de uma cidade do interior de Minas Gerais: os cidadãos estão satisfeitos?", que avaliou a satisfação do usuário de cirurgias eletivas de hospitais de ensino de Uberaba-MG sob as cinco dimensões da qualidade ${ }^{(13)}$. A proposta do presente estudo foi aprofundar a análise e explorar os achados no que se refere à dimensão tangibilidade.

O estudo foi desenvolvido em duas instituições hospitalares de um município do interior de Minas Gerais, sendo um hospital público e um hospital mantido por instituição privada, mas que oferece atendimento ao SUS, portanto, a pesquisa foi realizada apenas com os clientes usuários do serviço público. Para fins da pesquisa, os hospitais foram identificados por letras, sendo Hospital A e Hospital B.

Hospital A: hospital geral, federal, de ensino, de grande porte, que oferece atendimento de alta e média complexidade. Possui atualmente 316 leitos ativos.

Hospital B: hospital geral, mantido por uma instituição privada, classificado como entidade beneficente sem fins lucrativos, de ensino, de médio porte, que oferece atendimento de alta e média complexidade. Possui atualmente 140 leitos ativos.

Ambos os hospitais oferecem atendimento regional aos 27 municípios que compõem a macrorregião e atendem à demanda de formação profissional ao oferecer oportunidades de ensino baseadas na tríade ensino, pesquisa e extensão.

A amostra foi calculada estatisticamente, considerando uma perda de amostragem de $20 \%$ (recusas em participar), o número final de tentativas de entrevista foi $n=285$. Obteu-se um tamanho amostral de 228 clientes que buscaram atendimento cirúrgico nos hospitais especificados durante o período de junho de 2013 a fevereiro de 2014. A amostra foi selecionada de forma aleatória, utilizando-se o método de amostragem aleatória sistemática proporcional. Como critérios de inclusão, foram considerados aptos a participar do estudo os clientes maiores de 18 anos, responsáveis legalmente, residentes no município, que apresentassem condições clínicas favoráveis para responderem os instrumentos de coleta de dados e os que estivessem aguardando internação em sala de espera e fossem efetivamente internados, ou que estivessem internados há, no máximo, 24 horas para qualquer procedimento cirúrgico eletivo.

Já os critérios de exclusão foram clientes submetidos a procedimentos cirúrgicos de emergência, que fossem incapazes de responder ao questionário devido a condições clínicas, que evoluíram para óbito após o procedimento, e que não receberam alta hospitalar dentro do período de coleta de dados. A técnica utilizada para a coleta de dados foi a entrevista estruturada.

Primeiramente, a pesquisadora realizou reunião com o responsável técnico-administrativo de cada instituição, com o objetivo de apresentar o projeto e explanar os objetivos a serem alcançados. Após a autorização das instituições, as cirurgias eletivas foram acompanhadas diariamente por meio do mapa cirúrgico fornecido pelas mesmas. Os clientes que constavam no mapa cirúrgico foram abordados na sala de espera da recepção da instituição antes de adentrarem para o procedimento. Aqueles que atenderam aos critérios de inclusão/exclusão, apresentaram interesse em participar e assinaram o Termo de Consentimento Livre e Esclarecido, foram entrevistados.

A mesma abordagem foi realizada aos clientes que constavam no mapa cirúrgico, mas que já se encontravam internados há no máximo 24 horas do procedimento por orientação médica. A entrevista foi aplicada antes que eles fossem submetidos ao procedimento cirúrgico na referida instituição e de cinco a dez dias após a alta hospitalar, em suas residências, visto que no momento da primeira entrevista solicitaram-se aos clientes seus contatos residenciais e a autorização da visita. Os sujeitos deveriam participar dos dois momentos da pesquisa.

Para caracterização da amostra, foi aplicado um questionário sociodemográfico, cujo objetivo era traçar o perfil epidemiológico da amostra estudada, construído com base na revisão de literatura e atentando-se para os fatores cujas evidências apontam algum tipo de influência na satisfação. As variáveis de caracterização do entrevistado foram: instituição, idade, sexo, renda, estado civil, anos de estudo e situação conjugal. Já as variáveis de caracterização do procedimento cirúrgico foram: especialidade cirúrgica, porte cirúrgico, e tempo de internação. Consideraram-se quatro portes cirúrgicos: menor porte, porte intermediário, maior porte, maior porte $+^{(14)}$. 
A satisfação foi avaliada por meio do instrumento baseado no SERVQUAL, derivado do modelo de falha de serviços para avaliação da qualidade dos serviços de saúde e validado no Brasil(15). Constituise em uma escala multidimensional composta por duas subescalas: escala de expectativas e escala de percepção.

O instrumento avalia cinco dimensões da qualidade: tangibilidade (aparência do pessoal, estrutura física e informação disponível), confiabilidade (habilidade em prestar o serviço prometido com precisão e eficácia), responsividade (habilidade em ajudar e proporcionar o serviço rapidamente), segurança (habilidade em inspirar confiança e credibilidade) e empatia (habilidade em proporcionar atenção individual e especializada). Foi acrescentada, ainda, uma última questão, conforme instrumento validado, que visa avaliar a visão geral do cliente sobre a qualidade do serviço utilizado. Ao final do instrumento, utilizou-se ainda um questionário para alocação de pontos de acordo com a importância que cada dimensão da qualidade possui para os entrevistados. Nessa fase os entrevistados distribuíram 100 pontos entre as cinco dimensões, de acordo com a importância relativa que essas possuem para eles. Os itens de 1 a 4 da escala SERVQUAL são relativos à dimensão tangibilidade ${ }^{(15)}$. Cada escala é composta por 22 itens perfazendo um total de 44 itens, e esses são avaliados por escala LIKERT que apresenta pontuações variando de 1 (concordo totalmente) a 7 (discordo totalmente) ${ }^{(2)}$.

Os dados foram inseridos e armazenados em uma planilha eletrônica do programa Microsoft ${ }^{\circledR}$ Office Excel ${ }^{\oplus}$. Foram importados para o programa estatístico Statistical Package for Social Sciences (SPSS) versão 16.0 para processamento e análise.

Foram realizadas análises exploratórias (segundo estatística descritiva) por meio da distribuição de frequência absoluta e percentual para as variáveis qualitativas, e utilizadas as medidas descritivas de centralidade (média, mediana e posto médio) e de dispersão (desvio padrão, valor mínimo e valor máximo) para variáveis quantitativas. Para comparação entre expectativa e percepção para os itens da dimensão tangibilidade, foi utilizado teste $t$ pareado para variáveis qualitativas e Pearson para as quantitativas.

O projeto foi aprovado pelo Comitê de Ética em Pesquisa com Seres Humanos da Universidade Federal do Triângulo Mineiro, por meio do protocolo $\mathrm{n}^{\circ} 2527$, bem como pelas direções dos hospitais incluídos no estudo, conforme resolução $n^{\circ} 466 / 12$ sobre pesquisa envolvendo seres humanos ${ }^{(16)}$.

\section{- RESULTADOS}

Dos 228 clientes analisados, 163 (71,5\%) eram do hospital A e 65 (28,5\%) do Hospital B. Em relação à variável sexo, 40,8 \% eram do sexo masculino e 59,2 \% feminino. A faixa etária variou de 18 a 91 anos (média \pm desvio padrão: 48 $\pm 16,99$ ) e mediana de 49,5 anos. A média dos anos de estudo foi de 7,27 anos (média \pm desvio padrão: 7,27 \pm 4 ) e mediana de oito anos. Tratando-se da renda, $74(32,5 \%)$ ganhavam de uma dois salários mínimos, seguido de 60 (26,3\%) que recebiam de três a quatro salários. Já quanto à situação conjugal, casados representaram 99 (43,4\%) clientes da amostra e solteiros 61 (26,8\%).

O porte intermediário da cirurgia prevaleceu com 113 (49,6\%) clientes, seguido de 84 (36,8\%) do maior porte, $29(12,7 \%)$ do menor porte e dois $(0,6 \%)$ porte maior + . A especialidade cirúrgica mais frequente foi a ortopedia com 18,9\%. O tempo médio de internação foi de 3,11 dias (média \pm desvio padrão: $3,11 \pm 2,35)$ e uma mediana de três dias. Sendo que 13 clientes $(5,7 \%)$ tiveram o procedimento cirúrgico cancelado.

A análise da expectativa em relação ao domínio tangibilidade do instrumento SERVQUAL evidenciou que os clientes esperam que o hospital apresente estrutura física conservada e moderna, que a equipe tenha uma aparência pessoal adequada ao ambiente e que haja informação disponível de fácil compreensão. A maioria das respostas para os itens dessa dimensão foram 'concordo plenamente' e 'concordo' (Tabela 1). Para a expectativa, as médias de cada alternativa do domínio abordado variaram entre $6,43(s=0,64)$ e $6,90(s=0,33)$. 
Tabela 1 - Frequência de respostas dos participantes relacionada à expectativa para a dimensão Tangibilidade. Uberaba, MG, Brasil, 2014

\begin{tabular}{|c|c|c|c|c|c|c|c|}
\hline Tangibilidade & $\begin{array}{l}\text { DP* } \\
\text { n\% }\end{array}$ & $\begin{array}{c}\mathrm{D} \\
\mathbf{n} \%\end{array}$ & QD & Neutro & $\begin{array}{l}\text { QC } \\
\text { n\% }\end{array}$ & $\begin{array}{c}\mathbf{C} \\
\mathbf{n} \%\end{array}$ & $\begin{array}{l}\mathbf{C P} \\
\mathbf{n} \%\end{array}$ \\
\hline $\begin{array}{l}\text { 1. O hospital deve ter equipamentos } \\
\text { conservados e modernos. }(99,8)\end{array}$ & $\begin{array}{c}1 \\
(0,4) \\
\end{array}$ & $\begin{array}{c}1 \\
(0,4) \\
\end{array}$ & - & - & $\begin{array}{c}4 \\
(1,8) \\
\end{array}$ & $\begin{array}{c}26 \\
(11,4) \\
\end{array}$ & $\begin{array}{l}196 \\
(86)\end{array}$ \\
\hline $\begin{array}{l}\text { 2. As instalações físicas do hospital devem ser } \\
\text { visualmente bonitas e agradáveis. }\end{array}$ & $\begin{array}{c}2 \\
(0,9) \\
\end{array}$ & $\begin{array}{c}1 \\
(0,4)\end{array}$ & $\begin{array}{c}2 \\
(0,9) \\
\end{array}$ & $\begin{array}{c}14 \\
(6,1)\end{array}$ & $\begin{array}{c}11 \\
(6,1)\end{array}$ & $\begin{array}{c}42 \\
(18,4) \\
\end{array}$ & $\begin{array}{c}156 \\
(68,4) \\
\end{array}$ \\
\hline $\begin{array}{l}\text { 3. A equipe do hospital deve ter aparência } \\
\text { e trajes bem cuidados, de acordo com as } \\
\text { características do ambiente de trabalho. }(99,9)\end{array}$ & $\begin{array}{c}1 \\
(0,4)\end{array}$ & - & - & $\begin{array}{c}3 \\
(1,3)\end{array}$ & $\begin{array}{c}1 \\
(0,4)\end{array}$ & $\begin{array}{c}22 \\
(9,6)\end{array}$ & $\begin{array}{c}201 \\
(88,2)\end{array}$ \\
\hline $\begin{array}{l}\text { 4. Os relatórios e demais documentos } \\
\text { entregues ao paciente devem ser de fácil } \\
\text { compreensão e visualmente atrativos. }(99,9)\end{array}$ & - & - & - & $\begin{array}{c}1 \\
(0,4)\end{array}$ & - & $\begin{array}{c}19 \\
(8,3)\end{array}$ & $\begin{array}{c}208 \\
(91,2)\end{array}$ \\
\hline
\end{tabular}

*DP-' discordo plenamente'; D- ' discordo'; QD-'quase discordo'; QC-'quase concordo'; C-'concordo'; CP-'Concordo plenamente'.

A expectativa em relação ao atendimento em geral no serviço, item 23 No hospital, a qualidade geral do atendimento em cirurgia deve ser satisfatória', percebe-se uma alta expectativa com a qualidade em geral, enfatizada pela ausência de itens de conotação negativa ou neutra na avaliação, ou seja, todos os participantes consideraram importante que o atendimento seja de qualidade, seis $(2,6)$ 'concordo' e $222(97,4 \%)$ 'concordo plenamente'.

Na avaliação da percepção, a maioria dos clientes apresentou respostas positivas, ou seja, para estes entrevistados os hospitais atendem aos critérios relacionados à estrutura física, aparência pessoal e informações disponíveis. A maioria dos itens foi classificada com 'concordo plenamente' e 'concordo' (Tabela 2). As médias de cada item para percepção variaram entre $6,37(s=0,89)$ e $6,78(s=0,46)$. $\mathrm{Na}$ avaliação da percepção da qualidade geral, item 23 No hospital, a qualidade geral do atendimento em cirurgia é satisfatória', os participantes demonstraram boa percepção, atestando que a qualidade geral do atendimento é satisfatória, uma vez que não ocorreu nenhuma avaliação de conotação neutra ou negativa, $11(4,8)$ 'quase concordo', $39(17,1)$ 'concordo' e $178(78,1)$ 'concordo plenamente'.

Tabela 2 - Frequência de respostas dos participantes relacionada à percepção para a dimensão Tangibilidade. Uberaba, MG, Brasil, 2014

\begin{tabular}{|c|c|c|c|c|c|c|c|}
\hline \multirow[t]{2}{*}{ Tangibilidade } & DP* & D & QD & Neutro & QC & C & CP \\
\hline & $\mathrm{n} \%$ & $\mathrm{n} \%$ & $\mathrm{n} \%$ & $\mathbf{n} \%$ & $\mathrm{n} \%$ & $\mathrm{n} \%$ & $\mathrm{n} \%$ \\
\hline $\begin{array}{l}\text { 1. O hospital possui equipamentos } \\
\text { conservados e modernos. }\end{array}$ & $\begin{array}{c}1 \\
(0,4)\end{array}$ & $\begin{array}{c}1 \\
(0,4)\end{array}$ & $\begin{array}{c}2 \\
(0,9)\end{array}$ & $\begin{array}{c}4 \\
(1,8)\end{array}$ & $\begin{array}{c}17 \\
(7,5)\end{array}$ & $\begin{array}{c}51 \\
(22,4)\end{array}$ & $\begin{array}{c}152 \\
(66,7)\end{array}$ \\
\hline $\begin{array}{l}\text { 2. As instalações físicas do hospital são } \\
\text { visualmente bonitas e agradáveis. }(100,3)\end{array}$ & $\begin{array}{c}1 \\
(0,4)\end{array}$ & - & $\begin{array}{c}3 \\
(1,3)\end{array}$ & $\begin{array}{c}3 \\
(1,3)\end{array}$ & $\begin{array}{c}27 \\
(11,8)\end{array}$ & $\begin{array}{c}64 \\
(28,1)\end{array}$ & $\begin{array}{l}130 \\
(57)\end{array}$ \\
\hline $\begin{array}{l}\text { 3. A equipe do hospital tem aparência e } \\
\text { trajes bem cuidados, de acordo com as } \\
\text { características do ambiente de trabalho. }\end{array}$ & - & - & - & $\begin{array}{c}1 \\
(0,4)\end{array}$ & $\begin{array}{c}2 \\
(0,9)\end{array}$ & $\begin{array}{c}43 \\
(18,9)\end{array}$ & $\begin{array}{c}182 \\
(79,8)\end{array}$ \\
\hline $\begin{array}{l}\text { 4. Os relatórios e demais documentos } \\
\text { entregues ao paciente são de fácil } \\
\text { compreensão e visualmente atrativos. }(100,1)\end{array}$ & - & - & $\begin{array}{c}3 \\
(1,3)\end{array}$ & $\begin{array}{c}2 \\
(0,9)\end{array}$ & $\begin{array}{c}11 \\
(4,8)\end{array}$ & $\begin{array}{c}32 \\
(14)\end{array}$ & $\begin{array}{c}180 \\
(78,9)\end{array}$ \\
\hline
\end{tabular}

*DP-' discordo plenamente'; D- ' discordo'; QD-'quase discordo'; QC-'quase concordo'; C-'concordo'; CP-'Concordo plenamente'. 
O gap é a diferença entre a percepção e a expectativa e indica presença ou ausência de satisfação, onde gap positivo indica satisfação e o gap negativo insatisfação. Verificou-se que as médias do gap para os itens da tangibilidade variaram entre $-0,31(s=1,07)$ e $-0,05(s=0,75)$, indicando discreta insatisfação em relação aos objetos tangíveis.

$\mathrm{Na}$ análise dos dados quantitativos, foram comparadas as variáveis idade, anos de estudo e tempo de internação com o gap e utilizou-se a correlação de Spearman. Não houve resultado estatístico significativo para idade e tempo de internação, $r=0,112(p=0,090)$ e $r=-0,069(p=0,301)$ respectivamente. A variável 'anos de estudo' exibiu uma correlação fraca, $r=-0,127(p=0,055)$ (Tabela 3).

Tabela 3 - Associação entre a dimensão tangibilidade do instrumento SERVQUAL e as variáveis idade, anos de estudo e período de internação. Uberaba, MG, Brasil, 2014

\begin{tabular}{lccccccc} 
& \multicolumn{2}{c}{ Idade } & \multicolumn{2}{c}{ Anos de estudo } & \multicolumn{2}{c}{ Período de internação } \\
\hline Tangibilidade & $\mathrm{r}^{*}$ & $\mathrm{p}$ & $\mathrm{r}$ & $\mathrm{p}$ & $\mathrm{r}$ & $\mathrm{p}$ \\
\cline { 2 - 7 } & 0,112 & 0,09 & $-0,127$ & 0,055 & $-0,069$ & 0,301
\end{tabular}

${ }^{*} \mathrm{r}$ - coeficiente de correlação de Pearson; $p$ - valor de $p$.

Quanto às variáveis qualitativas instituição, cancelamento da cirurgia e gênero, não houve um resultado significativo empregando o teste teste t pareado (Tabela 4).

Tabela 4 - Associação entre a dimensão tangibilidade do instrumento SERVQUAL e as variáveis instituição, cancelamento da cirurgia e gênero empregando o teste t pareado. Uberaba, MG, Brasil, 2014

\begin{tabular}{lllcccc} 
& & & $\mathbf{n}$ & $\mathbf{x}$ & $\mathbf{s}$ & $\mathbf{p}$ \\
\cline { 2 - 7 } & Instituição & Hospital A & 164 & $-0,116$ & 0,684 & \\
\cline { 2 - 7 } Tangibilidade & Hospital B & 64 & $-0,270$ & 0,637 & 0,112 \\
\cline { 2 - 7 } & Cancelamento & Sim & 13 & $-0,096$ & 0,485 & \\
\cline { 2 - 7 } & \multirow{2}{*}{ Gênero } & Não & 215 & $-0,163$ & 0,683 & 0,73 \\
\cline { 2 - 7 } & & Feminino & 135 & $-0,143$ & 0,677 & \\
\cline { 2 - 7 } & Masculino & 93 & $-0,183$ & 0,670 & 0,659
\end{tabular}

*n - frequência; $x$ - média; s - desvio padrão; $p$ - valor de p.

\section{DISCUSSÃO}

Os resultados obtidos nesta pesquisa evidenciaram na população estudada, em relação aos aspectos sociodemográficos, maior porcentagem do gênero feminino. Um estudo realizado em um hospital regional de Londrina com objetivo de caracterizar o perfil de pacientes cirúrgicos verificou que $55 \%$ dos pacientes eram do sexo feminino ${ }^{(17)}$. Outro realizado em um hospital universitário, entrevistou 100 pacientes cirúrgicos e também houve predominância do sexo feminino $(81 \%)^{(18)}$. A mulher valoriza mais o cuidado com a própria saúde em relação ao homem, ainda as diferenças entre os sexos em relação à percepção de saúde são importantes determinantes da conduta do indivíduo que procura o sistema de saúde ${ }^{(19)}$.

A faixa etária predominante foi de 48 anos. A renda e escolaridade caracterizou baixo nível socioeconômico, refletindo as condições sociais da comunidade atendida pelas instituições. Corroborando com um estudo, em que a maioria possuía renda familiar de 1 a 3 salários mínimos ${ }^{(7)}$ e com outro realizado no hospital de Londrina onde houve uma média de idade de 35,3 anos evidenciando uma população adulta. 
Já em relação ao porte da cirurgia realizada pelo cliente, a maioria dos procedimentos são de porte intermediário e maior, sendo explicado pelas características de instituição de atenção à saúde de alta complexidade. A especialidade que mais esteve presente na caracterização dos procedimentos cirúrgicos foi a ortopedia. Em estudo realizado no hospital regional de Londrina com 110 pacientes cirúrgicos, a distribuição das especialidades foi: 27 (25\%) ortopédicas, 26 (24\%) gerais, 25 (23\%) vasculares, otorrinolaringologia 20 (18\%), infantil oito $(7 \%)$ e ginecológicas quatro $(4 \%)^{(17)}$.

A percepção dos clientes em relação à qualidade dos serviços de saúde tem alcançado um papel muito importante para realização de melhorias nos serviços. Incluí-lo neste processo confere reconhecimento e um atendimento humanizado. A busca por um atendimento de excelência deve ser pautada na assistência realizada por uma equipe interdisciplinar, que seja capaz de solucionar os problemas em saúde de forma humanizada ${ }^{(12)}$.

Para qualidade no processo de atenção, o local onde é oferecido o cuidado de saúde deve ser um ambiente limpo, com mobílias e equipamentos conservados, e equipe com trajes adequado, proporcionando, assim, um ambiente acolhedor e confortável ${ }^{(7-9)}$.

Os clientes demonstraram alta expectativa em relação às tecnologias duras do serviço e esperavam que essas influenciassem positivamente na qualidade da assistência prestada. A avaliação foi positiva quanto à apresentação dos aparelhos e da equipe. Já quanto às instalações físicas, o índice de resposta neutra foi maior. Pode-se inferir que, para alguns participantes, esse pode não ser um fator tão importante na avaliação, em relação aos outros.

O item quatro, "Os relatórios e demais documentos entregues ao paciente são de fácil compreensão e visualmente atrativos", foi o de maior destaque, sugerindo que os participantes valorizam a apresentação de documentos e relatórios de fácil entendimento. Não houve respostas negativas nessa alternativa. Já quanto "As instalações físicas do hospital devem ser visualmente bonitas e agradáveis", houve algumas conotações negativas, porém no geral a avaliação foi boa, assim como os itens 1 e 3 " $O$ hospital deve ter equipamentos conservados e modernos" e "A equipe do hospital deve ter aparência e trajes bem cuidados, de acordo com as características do ambiente e trabalho". Em relação ao atendimento geral no serviço, os clientes demonstraram alta expectativa, destacada pela ausência de conotação neutra e negativa. Um estudo iraniano evidenciou que entre as dimensões do SERVQUAL, a que apresentava maior expectativa era a Tangibilidade ${ }^{(20)}$. Sendo este item considerado importante para um atendimento de alto padrão.

A avaliação da percepção demonstrou que o hospital atende às questões relacionadas a este domínio. Um resultado positivo foi encontrado no que se refere à presença de equipamentos modernos e conservados, onde a conotação negativa foi mínima. A aparência física e dos profissionais foi satisfatória. Os documentos foram considerados compreensíveis e de boa apresentação. As respostas para a percepção da qualidade geral demonstraram boa percepção, não houve classificação neutra ou negativa, considerando assim o atendimento satisfatório. Estudo realizado em um hospital privado de São Paulo mostrou que os atributos que mais inteferiram na insatisfacação dos participantes foram qualidade do sabor da refeição 54 (76\%), instalações físicas do quarto 56 (78,9\%) e tempo de espera no atendimento incial 59 (83,1\%), evidenciando que a aparência da estrutura possui influência sobre a qualidade $^{(21)}$.

Entretanto, quando o gap foi analisado, esse mostrou que os clientes estavam discretamente insatisfeitos com o serviço, ou seja, a percepção não superou a expectativa. No entanto, as médias para cada item em relação à expectativa e percepção demonstraram níveis altos em uma escala de sete pontos e, quando comparados entre si, o gap apesar de negativo esteve próximoa zero. Contudo, é relevante que os gestores em saúde busquem melhorias para alcançar níveis mais elevados de satisfação do cliente.

Uma pesquisacom clientes em tratamento dialítico apresentou resultado semelhante em relação ao gap (-0.29 \pm 0.51$)$, porém o estudo justificou que apesar de negativo, não era tão discrepante a diferença, considerando aceitável a qualidade do serviço, porém esforços são necessários para alcançar o nível ideal ${ }^{(22)}$.

Não houve correlação significativa entre o gap e a idade, tempo de internação, instituição, cancelamento e gênero. As dimensões da qualidade do serviço em outra pesquisa também não 
apresentaram associação significativa com sexo, idade, estado civil e nível de educação, corroborando com os dados apresentados ${ }^{(19)}$. Estudo analisando a tangibilidade em atenção primária $(\mathrm{n}=319)$, ao associar a variável ambiente físico com a idade dos participantes, encontrou aprovação de 38 (92,70\%), entre o público idoso(7).

Na pesquisa aqui apresentada, anos de estudo apresentou uma correlação fraca, já investigação realizada com pacientes renais crônicos não encontrou associação com esta variável, entretanto encontrou diferença entre os níveis de escolaridade. As expectativas dos indivíduos se tornaram mais razoáveis ao aumentar seu nível de educação e suas expectativas foram diminuídas pelo aumento do conhecimento e da conscientização dos processos de tratamento ${ }^{(22)}$.

A qualidade avaliada a partir dos objetos tangíveis é mais fácil de ser detectada pelo cliente, possibilitando intervenções concretas ${ }^{(21)}$. Entretanto, objetos tangíveis são de menor relevância para o usuário.

O estudo teve como limitações o cumprimento do calendário de cirurgias e a localização dos clientes, devido a obras na instituição e fase de transição administrativa. Ainda, muitos pacientes foram excluídos da amostra por não se encaixarem nos critérios de inclusão/exclusão principalmente em relação à procedência e à gravidade dos casos cirúrgicos. Um ponto forte foi a averiguação da abordagem do tema em relação à tangibilidade, fato esse pouco investigado na literatura científica.

\section{- CONCLUSÃO}

No estudo, a dimensão tangibilidade indicou insatisfação, ou seja, não atendeu às expectativas dos participantes em relação aos objetos tangíveis. No entanto, houve poucas avaliações negativas tanto quanto à expectativa quanto à percepção, caracterizando que subsídios tangíveis são necessários para conferir qualidade ao serviço. No geral, tanto a avaliação da expectivativa quanto da perccepção da qualidade geral do hospital foram satisfatórias.

Visto que a qualidade, sob a ótica do usuário, é um método para instituir mudanças no âmbito da saúde pelos gestores e profissionais de saúde, espera-se que estudo possa incentivar e contribuir para criação de ações de melhorias no serviço de saúde, assegurando uma assistência ao paciente de qualidade.

\section{REFERÊNCIAS}

1. Cabello E, Chirinos JL. Validación y aplicabilidad de encuestas SERVQUAL modificadas para medir la satisfacción de usuarios externos en servicios de salud. Rev Med Hered.[Internet] 2012;23(2) [acesso em 29 mai 2016].Disponível: http://www.scielo.org.pe/scielo.php?script=sci_arttext\&pid=S1018-130X2012000200003\&lng= es\&nrm=iso.

2. Parasuraman A, Berry LL, ZeithamIVA. SERVQUAL: a multiple-item scale for measuring consumer perceptions of service quality. J Retailing. 1988;64(1):12-40.

3. Calixto-Olalde MG, Sawada NO, Hayashida M, Mendes IAC, Trevizan MA, de Godoy S. Escala SERVQUAL: validación em población mexicana. Texto Contexto Enferm. [Internet] 2011;20(3) [acesso em 29 mai 2016]. Disponível:. http://dx.doi.org/10.1590/S0104-07072011000300014.

4. Ricci NA, Wanderley FS, de Oliveira MS, Rebelatto JR. O hospital-escola de São Carlos: análise do funcionamento por meio da satisfação dos usuários. Ciênc. saúde coletiva. [Internet] 2011;16(Suppl 1) [acesso em 29 mai 2016]. Disponível:http://dx.doi.org/10.1590/S1413-81232011000700044.

5. Parasuraman A, Zeithaml VA, Berry LL. A conceptual model of service quality and its implications for future research. J Marketing. [Internet] 1985;(49) [acesso em 29 mai 2016]. Disponível: https://www.researchgate.net/ publication/225083670_A_Conceptual_Model_of_Service_Quality_and_its_Implication_for_Future_Research_ SERVQUAL.

6. Ferreira ABH. Dicionário Aurélio Básico da Língua Portuguesa. Rio de Janeiro: Positivo; 2010. 
7. Dias OV, Vieira MA, Dias JP, Ramos LH. As dimensões da satisfação dos usuários do Programa Saúde da Família: confiabilidade e empatia. Acta paul.enferm. [Internet] 2011;24(2) [acesso em 29 mai 2016]. Disponível: http:// dx.doi.org/10.1590/S0103-21002011000200011.

8. Ministério da Saúde (BR).Secretaria de Atenção à Saúde. Núcleo Técnico da Política Nacional de Humanização. Ambiência. $2^{a}$ ed. Brasília: Ministério da Saúde; 2010.

9. Ministério da Saúde (BR). Secretaria-Executiva. Núcleo Técnico da Política Nacional de Humanização. Política Nacional de Humanização. Brasília: Ministério da Saúde; 2013.

10. dos Santos ALS, dos Santos RAA, Carmo AFS, Gusmão-Filho FAR, Mendes RNC. Avaliação da qualidade: satisfação dos usuários de unidades de terapia intensiva pediátrica mista e obstétrica. J. res.: fundam. care. online. [Internet] 2015;7(3) [acesso em 29 mai 2016]. Disponível: http://dx.doi.org/10.9789/2175-5361.2015.v7i3.2974-2984.

11. Lyu H, Wick EC, Housman M, Freischlag JA, Makary MA. Patient Satisfaction as a Possible Indicator of Quality Surgical Care. JAMA Surg. [Internet] 2013;148(4) [acesso em 29 mai 2016]. Disponível: http://dx.doi. org/10.1001/2013.jamasurg.270.

12. de Freitas JS, Silva AEBC, Minamisava R, Bezerra ALQ, de Sousa MRG. Quality of nursing care and satisfaction of patients attended at a teaching hospital. Rev. Latino-Am. Enfermagem.[Internet] 2014;22(3) [acesso em 30 mai 2016]. Disponível: http://dx.doi.org/10.1590/0104-1169.3241.2437.

13. Tirones ASD. Cirurgias eletivas em hospitais públicos de uma cidade do interior de minas gerais:os cidadãos estão satisfeitos?[dissertação]. Uberaba(MG):Universidade Federal do Triângulo Mineiro; 2014.

14. Santos JS, Kemp R. Fundamentos básicos para a cirurgia e cuidados perioperatórios. Medicina.[Internet] 2011;44(1) [acesso em 30 mai 2016]. Disponível: http://dx.doi.org/10.11606/issn.2176-7262.v44i1p2-17.

15. Borges JBC, de Carvalho SMR, Silva MAM. Qualidade do serviço prestado aos pacientes de cirurgia cardíaca do Sistema Único de Saúde-SUS. Rev Bras Cir Cardiovasc. 2010;25(2) [acesso em 29 mai 2016]. Disponível: http:// dx.doi.org/10.1590/S0102-76382010000200008.

16. Ministério da Saúde (BR). Conselho Nacional de Saúde. Diretrizes e normas regulamentadoras de pesquisa envolvendo seres humanos. Resolução n. 466, de 12 de dezembro de 2012. Brasília; 2012.

17. Giordani AT, Sonobe HM, Ezaias GM, Valério MA, Barra MR, Stadler DV. Perfil de pacientes cirúrgicos atendidos em um hospital público. Rev enferm UFPE on line. [Internet] 2015;9(1) [acesso em 31 mar 2017]. Disponível: https://periodicos.ufpe.br/revistas/revistaenfermagem/article/view/10306.

18. dos Santos MA, Rossi LA, Paiva L, Dantas RAS, Pompeo DA, Machado ECB. Medida da ansiedade e depressão em pacientes no pré- operatório de cirurgias eletivas. Rev. Eletr. Enf. [Internet] 2012;14(4) [acesso em 31 mar 2017 ]. Disponível:https://www.fen.ufg.br/fen_revista/v14/n4/pdf/v14n4a21.pdf.

19. Werneck J. Intersecções de raça/etnia, gênero e classe: faces cotidianas e teóricas. Entrelugares e mobilidades: desafios feministas. Tubarão: Copiart; 2014.

20. Zarei A, Arab M, Froushani AR, Rashidian A, Tabatabaei SMG. Service quality of private hospitals: the Iranian patients' perspective. BMC Health Serv Res.[Internet] 2012;(12) [acesso em 29 mai 2016]. Disponível: http://dx.doi. org/10.1186/1472-6963-12-31.

21. da Cruz WBS, Melleiro MM. Análise da satisfação dos usuários de um hospital privado. Rev. esc. enferm. USP. [Internet] 2010;44(1) [acesso em 29 mai 2016]. Disponível:http://dx.doi.org/10.1590/S0080-62342010000100021.

22. Bahadori M, Raadabadi M, Jamebozorgi MH, Salesi M, Ravangard R. Measuring the quality of provided services for patients with chronic kidney disease. Nephro Urol Mon. 2014;6(5) [acesso em 29 mai 2016]. Disponível: http:// dx.doi.org/10.5812/numonthly. 21810 . 